\title{
ÁLGEBRA LINEAR NA ENGENHARIA: REFLEXÕES A PARTIR DA VISÃO DE PROFESSORES
}

DOI: 10.37702/2175-957X.COBENGE.2021.3482

Juliana Martins Philot - juliana.philot@maua.br

Instituto Mauá de Tecnologia

Rua Bélgica 719

09210-030 - Santo André - SP

Barbara Lutaif Bianchini - barbaralb@gmail.com

Pontifícia Universidade Católica de São Paulo

Rua Frei Vicente do Salvador 183

02019-000 - São Paulo - SP

Eloiza Gomes - eloiza@maua.br

IMT

Rua Manuel Figueiredo Landim 126

04693-130 - São Paulo - SP

Resumo: Pesquisas em Educação Matemáticas apontam para a importância da Álgebra Linear em vários cursos de graduação, em especial a Engenharia. É consenso entre os pesquisadores dessa área que os estudantes apresentam dificuldades no entendimento dos conteúdos abordadas em Álgebra Linear devido ao formalismo inerente a este assunto e a falta de maturidade matemática dos alunos no momento em que essa disciplina é ministrada nos cursos. Um aspecto que está ressaltado nas Diretrizes Curriculares Nacionais do Curso de Graduação em Engenharia é que os cursos de Engenharia não podem mais serem vistos como um conjunto de conhecimentos técnicos, ou não técnicos, isolados, isto aponta que a contextualização dos assuntos deve ser privilegiada. Nesse sentido, o objetivo desse artigo é apresentar um recorde de uma pesquisa de doutorado que tem como um de seus focos elaborar situações de aprendizagem contextualizadas para o ensino de autovalor e autovetor para cursos de Engenharia. Apresenta-se a análise de um questionário respondido por professores de disciplinas específicas de cursos de Engenharia de uma Instituição de Ensino Superior, na perspectiva de entender quais os conteúdos de Álgebra Linear são mais explorados. Percebe-se que todos os entrevistados utilizam em suas aulas o objeto matemático sistemas lineares e que os assuntos mais específicos, como autovalor e autovetor estão 


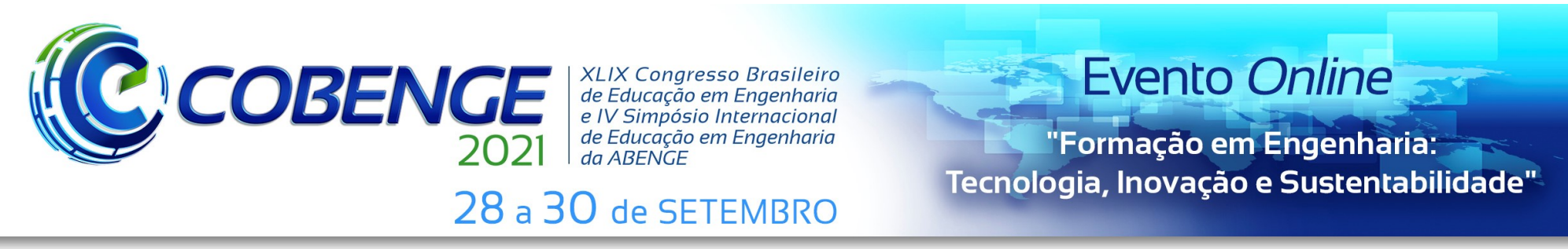

presentes em disciplinas ministradas no final do curso.

Palavras-chave: Álgebra Linear na Engenharia, Entrevista com professores 


\section{INTRODUÇÃO}

Pesquisas em Educação Matemática (NOMURA (2014), PRADO (2016), FONTENELLE (2018)) têm apontado a importância de se estudar os conceitos de Álgebra Linear em vários cursos de graduação, devido à interdisciplinaridade desta com outros ramos da Matemática e até mesmo com outras disciplinas. Prado (2016) relata que os estudantes apresentam dificuldades no entendimento do conteúdo de Álgebra Linear devido ao formalismo inerente a este assunto e a falta de maturidade Matemática dos alunos no momento em que essa disciplina é ministrada nos cursos de graduação, sendo esses identificados como alguns dos principais entraves.

Nessa mesma linha, Celestino (2000) relata que pesquisas sobre o ensino e a aprendizagem de Álgebra Linear são relativamente recentes, e que nos Estados Unidos esses estudos iniciaram por volta de 1990, com a criação do Grupo Linear Algebra Curriculum Study Group (LACSG). O mesmo autor menciona que, simultaneamente ao que acontecia nos EUA, na França, Jean Luc Dorier publicava sua tese sobre este assunto e, por volta de 1997, um grupo de uma universidade situada na cidade de Campinas-SP começou a difundir este assunto pelo Brasil.

Philot (2020) realizou um levantamento bibliográfico sobre as teses e dissertações que abordam o tema Álgebra Linear no Ensino Superior no Brasil publicados entre os anos de 2011 e 2018, com o objetivo de identificar as principais tendências das pesquisas realizadas e as lacunas não investigadas e constatou que embora exista um número significativo de trabalhos com esse tema, muitos deles conduzem suas pesquisas nos cursos de Licenciatura e Bacharelado em Matemática.

Além disso, Ferruzzi e Almeida (2013) discutem que não podemos mais ministrar disciplinas de Matemática de forma isolada, sem conexão com as disciplinas específicas, conforme destacam:

[...] entende-se ser importante conceber a Matemática como uma ciência que pode ser praticada, vivenciando-a de forma que alguns problemas que surjam do cotidiano do estudante possam ser trabalhados em sala de aula, possibilitando a exploração e construção dos conceitos matemáticos por meio de atividades que tenham significado para o aluno, buscando assim a construção do seu conhecimento. (FERRUZZI; ALMEIDA, 2013, p.157).

Estudos, como por exemplo, de Firmino e Siqueira (2017) apontam que o ensino de Matemática para estudantes de Engenharia ainda é lecionado de maneira tradicional, sem contextualização, sem relação com as disciplinas específicas e com sua atuação profissional. Ferruzzi e Almeida (2013) também indicam a ausência de livros e materiais didáticos que possam auxiliar o professor de Matemática que leciona nos cursos de Engenharia a ter uma atuação mais próxima da que se espera.

Outro aspecto que vem sendo ressaltado nas Diretrizes Curriculares Nacionais do Curso de Graduação em Engenharia (DCN) (BRASIL, 2019) é que os cursos de Engenharia não podem mais serem vistos como um conjunto de conhecimentos técnicos, ou não técnicos, isolados. A formação em Engenharia deve ser vista como um processo que envolve pessoas, necessidades, expectativas, comportamentos, além de técnicas e conteúdos.

Ainda nesse sentido, Ribeiro (2018) enfatiza que o ensino de conteúdos de Álgebra Linear de forma desvinculada pode contribuir para as dificuldades dos processos de ensino e de aprendizagem desta. Camarena (2017) destaca a importância da contextualização, neste sentido, afirma que: 
[...] é importante porque um conceito matemático contextualizado adquire significado por meio das contextualizações realizadas para a apresentação de um determinado assunto, porque os conceitos não são isolados, são constituídos sob a forma de uma rede e mantêm relações entre eles (CAMARENA, 2000; MURO, 2004; TREJO, 2005 apud CAMARENA, 2017, p.11, tradução nossa).

Pensando na formação de um engenheiro com essas características, utilizamos o Modelo Didático da Matemática em Contexto (MoDiMaCo) que faz parte da fase didática da Teoria A Matemática no Contexto da Ciência (TMCC), elaborada pela pesquisadora Patrícia Camarena, que relataremos brevemente mais adiante. O MoDiMaCo tem como ideia principal estimular a construção do conhecimento por parte do aluno e o desenvolvimento de habilidades para a transferência do conhecimento matemático para as suas futuras áreas de atuação profissional e para a sua vida social. Tal modelo emprega como estratégias de ensino eventos contextualizados que, segundo Camarena (2013a), são problemas ou projetos que desempenham o papel de integrar as disciplinas matemáticas e não matemáticas, convertendo-os em ferramentas para o trabalho interdisciplinar no ambiente de aprendizagem.

Diante deste cenário, o objetivo do trabalho de doutorado, que está sendo desenvolvido pela primeira autora deste artigo, é elaborar um evento contextualizado voltado à construção dos conceitos de autovalor e autovetor e aplicar estes como uma ferramenta voltada ao ensino, estando assim em concordância ao MoDiMaCo e com as DCN (BRASIL, 2019), e analisar quais suas possíveis contribuições, do ponto de vista cognitivo, para o futuro engenheiro. Para alcançarmos esse objetivo geral, precisamos atingir alguns objetivos específicos, sendo um desses realizar entrevistas com professores de disciplinas específicas dos cursos de Engenharia de uma Instituição de Ensino Superior (IES) do Estado de São Paulo. Tais entrevistas foram realizadas no período de novembro de 2019 a março de 2020, um dos objetivos dessa entrevista é o de obter um panorama da utilização da Álgebra Linear nos cursos de Engenharia, sob o olhar dos professores entrevistados, para posteriormente em conjunto com o evento contextualizado auxiliar na preparação de uma atividade com conteúdos de Álgebra Linear.

Nesse artigo, apresentamos um recorte de uma entrevista reflexiva, semiestruturada, e a preparação do roteiro e a conduta dessa entrevista baseou-se no livro "A Entrevista na Pesquisa em Educação: a prática reflexiva", e relataremos somente o primeiro momento da entrevista, denominada de contato inicial. trabalho.

A seguir, apresentamos o referencial teórico-metodológico que foi empregado neste

\section{A MATEMÁTICA NO CONTEXTO DA CIÊNCIA}

A TMCC é uma teoria educacional que se iniciou em 1982, no Instituto Politécnico Nacional do México, segundo Camarena (2013a) de reflexões sobre: o objetivo do curso de graduação no qual a Matemática está sendo ensinada; como motivar o estudante desse curso a aprender Matemática; como vincular essa ciência com a futura profissão do graduando; como a Matemática pode contribuir com a formação integral do estudante e apoiar o desenvolvimento de competências profissionais.

Essa teoria tem como um dos principais objetivos retratar aspectos sobre o ensino da Matemática em cursos de graduação nos quais não visam a formação de matemáticos, mas sim uma formação holística e integral do aluno, baseando-se, segundo Camarena (2013a) nos seguintes paradigmas: a Matemática é uma ferramenta de apoio ou disciplina 
formativa para os profissionais; a Matemática tem uma função específica no nível universitário; os conhecimentos nascem integrados.

Camarena $(2014,2017)$ considera o ambiente de ensino e de aprendizagem constituído por um sistema complexo, no qual estão presentes os conteúdos a ensinar, o estudante e o professor, bem como as interações que ocorrem entre eles. Essas interações são compostas por cinco fases que interagem entre si denominadas: fase curricular (iniciou em 1984), fase didática (iniciou em 1987), fase epistemológica (iniciou em 1988), fase docente (iniciou em 1990) e fase cognitiva (iniciou em 1992). Esse sistema também é influenciado por fatores do tipo econômico, cultural, emocional, social e político.

A fase curricular da TMCC, tem como objetivo principal elaborar um currículo de Matemática para cursos de graduação no qual esta ciência está a serviço. Para tanto, foi desenvolvida nessa fase, uma metodologia denominada Dipcing (Diseño de programas de estudio de matemáticas em carreras de ingeniería) (CAMARENA, 2002), para projetar programas de estudo, inicialmente de Matemática para cursos de Engenharia e mais tarde, para outros cursos no qual esta ciência está à serviço e posteriormente estendida para outras Ciências Básicas.

Essa metodologia se fundamenta no paradigma educacional de que o currículo de Matemática proposto para um curso de graduação, que não visa a formação de matemático, tem que ser estruturado de forma clara e objetiva para atender às necessidades do curso no qual foi proposto, fornecendo as ferramentas necessárias para a formação do aluno. Além do fato de que a Matemática tem que ser formativa para o estudante, isto é, pode conter conteúdos que não são usados diretamente nos cursos, mas que auxiliam na compreensão dos conceitos que são fundamentais para a formação matemática e entendimento de seus conceitos que o estudante irá aplicar nas disciplinas específicas e, provavelmente em sua carreira, conforme ressaltam os artigos de Camarena (2002, 2009, 2013a).

Para cumprir com tais premissas, a metodologia Dipicing, segundo Camarena (2002), é organizada em três etapas: etapa central, etapa precedente e etapa consequente. A etapa central tem como objetivo principal apontar conteúdos matemáticos que são abordados implícita ou explicitamente nas disciplinas de Ciências Básicas (Biologia, Física e Química), nas disciplinas específicas e nas disciplinas profissionalizantes para cada uma das habilitações dos cursos de Engenharia.

Uma vez definido quais os conteúdos matemáticos são importantes para determinado curso, precisa-se investigar o que o ingressante precisa conhecer para conseguir acompanhar de maneira satisfatória esse curso. A investigação de quais os prérequisitos necessários e como atingi-los é realizada na etapa precedente. Para completar a elaboração de um currículo que atenda às necessidades profissionais e laborais de egresso é necessária a vinculação entre os conteúdos matemáticos presentes no curso de graduação com os conteúdos utilizados no cotidiano do profissional da área em que se está construindo o currículo. Dessa forma, começam as investigações com profissionais que estão na ativa, para compor a etapa consequente.

Depois de criada a fase curricular e da aplicação da metodologia Dipcing, começam a ter-se discernimento sobre a utilização da Matemática na Engenharia, compreendendo onde se utiliza, como se utiliza, entre outras questões, o que acaba acarretando outros questionamentos. Por exemplo, como ensinar conteúdos de Matemática que estão contextualizados em um problema de Engenharia, como trabalhar com os alunos essas interdisciplinaridades, como desenvolver no aluno habilidades para que ele consiga transferir seus conhecimentos matemáticos para as suas futuras áreas de atuação profissional e para a sua vida social. Essas indagações deram origem à fase didática e o Modelo Didático da Matemática no Contexto (MoDiMaCo) que, como já mencionamos 
anteriormente, tem como objetivo principal estimular a construção do conhecimento e o desenvolvimento de habilidades, por parte dos graduandos tornando-o capaz de transferir o conhecimento matemático adquirido para suas futuras profissões e para sua vida social, por meio de eventos contextualizados.

Nessa mesma perspectiva, espera-se que mediante a aplicação deste modelo, o estudante perceba como a Matemática está vinculada a sua profissão, ficando mais motivado e dando mais significado aos conceitos estudados, gerando desta forma uma aprendizagem mais sólida e duradoura.

A fase epistemológica contribui no entendimento da interdisciplinaridade da Matemática com outras áreas de conhecimento e o porquê essas disciplinas estão sendo estudadas. Ainda nesse cenário, surge a preocupação de se ter um docente capacitado para trabalhar com este currículo, com a interdisciplinaridade dos conteúdos matemáticos nas disciplinas específicas dos cursos de Engenharia, na vida profissional e no cotidiano do estudante, dessas inquietudes surge a fase docente. Por fim, para explicar o funcionamento cognitivo da aprendizagem com o emprego da contextualização, surge a fase cognitiva. Descrevemos neste artigo de maneira suscinta a TMCC, mas por se tratar de uma teoria, todas as suas fases apresentam metodologias e fundamentação teórica de acordo com os paradigmas em que se baseiam.

Neste artigo utilizamos a etapa central, pois com a análise das entrevistas realizadas obtivemos informações a respeito dos conceitos/temas de Matemática que foram detectados na visão dos professores entrevistados. Na próxima seção relatamos os procedimentos metodológicos da pesquisa.

\section{PROCEDIMENTOS METODOLÓGICOS}

Este trabalho, como já foi ressaltado, é um recorte de uma entrevista reflexiva, semiestruturada que, segundo Szymanski, Almeida e Prandini (2018), não contém um roteiro fechado, pode-se fazer adequações baseadas na fala do entrevistado caso seja necessário. Esse tipo de entrevista segue um roteiro previamente preparado, podendo conter questões abertas e/ou fechadas seguindo uma conversa mais informal.

A preparação do roteiro e a conduta dessa entrevista baseou-se no livro "A Entrevista na Pesquisa em Educação: a prática reflexiva", no qual a entrevista está dividida em dois momentos. No primeiro momento é quando o entrevistador e o participante se conhecem melhor e, o segundo momento, é a condução da entrevista, sendo esta subdividida em 6 fases: aquecimento, questão desencadeadora, expressão da compreensão, sínteses, questões (de esclarecimento, focalizadoras e aprofundamento) e devolução. Nesse artigo abordaremos somente o primeiro momento, denominado pelas autoras de contato inicial.

Segundo Szymanski, Almeida e Prandini (2018, p.20) no contato inicial "seria desejável que ocorresse uma apresentação mútua entre o entrevistador e o entrevistado, e que se buscasse esclarecer a finalidade da pesquisa, abrir um espaço para perguntas e dúvidas, estabelecendo uma relação cordial".

Para atender os procedimentos citados, preparamos um formulário no Google Docs, que continha questões para caracterizar o professor e quatro questões sobre o tema de Algebra Linear. As questões referentes à caracterização de professores, eram mais pessoais e de formação acadêmica, como por exemplo: nome, data de nascimento, cargo que ocupa na instituição na qual estávamos pesquisando, formação acadêmica, atuação profissional, ano que se formou e ano que começou a lecionar. Muitas dessas respostas poderiam ser facilmente encontradas no Currículo Lattes, porém elas serviram como um primeiro contato, para entender um pouco mais sobre o sujeito da pesquisa. Das quatro 
questões propostas no formulário disponibilizado, as de número 3 e 4 referem-se diretamente à Álgebra Linear, na questão 4 foram apontados temas comumente trabalhados nesta disciplina, como forma de orientar os entrevistados. A seguir relatamos tais questões:

1. Em 2019, em quais habilitações do curso de Engenharia você atuou e quais disciplinas específicas lecionou?

2. Essas disciplinas que você atuou no ano de 2019 eram em quais séries?

3. Essas disciplinas utilizavam conceitos de Álgebra Linear?

4. Quais conteúdos dentre sistemas lineares, espaços vetoriais, dependência linear base e dimensão, transformação linear, autovalor e autovetor, diagonalização, produto interno, ortogonalização, entre outros, foram utilizados nas disciplinas?

Na seção seguinte, descrevemos o contato inicial da entrevista e as discussões e análise dos dados obtidos com esse primeiro momento.

\section{APRESENTAÇÃO, DISCUSSÃO E ANÁLISE DOS RESULTADOS}

Iniciamos essas entrevistas, por e-mail, com o contato inicial que, como vimos anteriormente, é o momento em que nos apresentamos, para que quando a entrevista presencial acontecesse, a conversa fosse um pouco mais informal, e nós estaríamos mais descontraídos, podendo fluir melhor a entrevista, o entrevistado estaria mais à vontade e possivelmente não omitiria nenhum dado que fosse importante para o nosso trabalho.

Nesse momento, a primeira autora deste artigo, entrou em contato via e-mail com 4 professores, identificando-se, informando em qual Instituição trabalhava, que estava cursando o doutorado na PUC-SP em Educação Matemática e que sua pesquisa tinha por objetivo elaborar eventos contextualizados voltados à construção de conceitos de autovalor e autovetor.

Neste e-mail, foi perguntado aos docentes se eles teriam interesse em participar do trabalho de pesquisa, caso a resposta fosse positiva, se, em uma segunda etapa poderíamos marcar uma entrevista presencial, com duração de aproximadamente 30 minutos. Neste primeiro momento, alertávamos os entrevistados que o trabalho estava de acordo com o Comitê de Ética da PUC-SP e que caso aceitassem participar, teriam que assinar o Termo de Consentimento Livre e Esclarecido (TCLE). Esse termo explica 0 objetivo da pesquisa, a importância da participação dos docentes, quais os benefícios e os riscos que poderiam ocorrer com a pesquisa, que todas as informações coletadas neste estudo seriam estritamente confidenciais. Também explicávamos que somente a pesquisadora e sua orientadora teriam conhecimento da identidade do professor e que estas se comprometiam em mantê-las em sigilo ao publicar os resultados desta pesquisa, garantindo que os dados seriam utilizados exclusivamente para fins didáticos e/ou científicos. Este termo informava que essa entrevista estava autorizada pelo Pró-Reitor Acadêmico da Instituição em que seria realizada e que o segundo momento, a entrevista presencial, seria gravada.

Se o entrevistado estivesse de acordo em participar, pedíamos que respondesse ao formulário do Google Docs que estava anexo no e-mail, e para finalizar perguntávamos ao professor qual seria o melhor dia e horário para realizar o segundo momento da entrevista que seria presencial, se tinham alguma dúvida e se queriam fazer alguma pergunta.

A seguir, reapresentamos as quatro questões do contato inicial das entrevistas, relatando as respostas dos professores, o objetivo dessas questões e quais as conclusões que obtivemos com essas respostas. Esse conjunto de quatro questões, que foram 
perguntadas no contato inicial, tinham como objetivo fornecer um panorama da utilização da Álgebra Linear nos cursos de Engenharia, sobre o olhar dos professores entrevistados, para mais tarde juntamente com o evento contextualizado, auxiliar na preparação de uma atividade com conteúdo de Álgebra Linear.

\section{Em 2019, em quais habilitações do curso de Engenharia você atuou e quais disciplinas específicas lecionou?}

Quadro 1: Habilitações que os professores respondentes da pesquisa atuavam

\begin{tabular}{|l|c|c|c|c|}
\hline Habilitações do curso de Engenharia & Professor 1 & Professor 2 & Professor 3 & Professor 4 \\
\hline Engenharia Civil & & & $\mathrm{X}$ & \\
\hline Engenharia da Computação & $\mathrm{X}$ & & & $\mathrm{X}$ \\
\hline Engenharia de Controle e Automação & $\mathrm{X}$ & $\mathrm{X}$ & & $\mathrm{X}$ \\
\hline Engenharia Eletrônica & $\mathrm{X}$ & & & $\mathrm{X}$ \\
\hline Engenharia Elétrica & $\mathrm{X}$ & $\mathrm{X}$ & & $\mathrm{X}$ \\
\hline Engenharia Mecânica & & & $\mathrm{X}$ & $\mathrm{X}$ \\
\hline
\end{tabular}

Fonte: dados da pesquisa

Pode-se observar que os professores entrevistados atuam nos cursos de Engenharia Civil, Engenharia da Computação, Engenharia de Controle e Automação, Engenharia Eletrônica, Engenharia Elétrica, Engenharia Mecânica. Focamos inicialmente, no nosso estudo, nos cursos de Engenharia da Computação, Engenharia de Controle e Automação, Engenharia Eletrônica, Engenharia Elétrica e Mecânica, pois essas habilitações foram as que mais apareceram na nossa amostra. Além dessa constatação, percebe-se que essas são habilitações que utilizam bastante a Matemática como ferramenta. Podemos depreender, a partir dos dados contidos no Quadro 1 que o professor 1 leciona em quatros habilitações diferentes e o professor 4 em cinco.

A segunda questão, informa-nos sobre as disciplinas e séries que esses professores atuam.

\section{Essas disciplinas que você atuou no ano de 2019 eram em quais séries?}

Quadro 2: Disciplinas e séries que os professores atuaram em 2019

\begin{tabular}{|l|c|c|c|c|c|}
\hline Disciplinas & Série & Professor 1 & Professor 2 & Professor 3 & Professor 4 \\
\hline Algoritmos e Programação & $1^{1^{\mathrm{a}}}$ & $\mathrm{X}$ & & & \\
\hline Sistemas de Controle I & $5^{\mathrm{a}}$ & $\mathrm{X}$ & & & \\
\hline Robótica Industrial & $5^{\underline{a}}$ & $\mathrm{X}$ & & & \\
\hline $\begin{array}{l}\text { Introdução a Aprendizagem de } \\
\text { Máquina }\end{array}$ & $5^{\underline{a}}$ & $\mathrm{X}$ & & & \\
\hline Robótica em Ambientes Virtuais & $5^{\underline{a}}$ & $\mathrm{X}$ & & & \\
\hline $\begin{array}{l}\text { Acionamentos e Instalações } \\
\text { Elétricas Industriais }\end{array}$ & $5^{\underline{a}}$ & & $X$ & & \\
\hline
\end{tabular}


28 a 30 de SETEMBRO

"Formação em Engenharia:

Tecnologia, Inovação e Sustentabilidade"

\begin{tabular}{|l|c|c|c|c|c|}
\hline Sistema de Potência & $4^{\underline{a}}$ & & X & & \\
\hline Conversão de Energia & $4^{\underline{a}}$ & & $\mathrm{X}$ & $\mathrm{X}$ & \\
\hline Projeto de Usinas e Subestações & $5^{\mathrm{a}}$ & & $\mathrm{X}$ & & \\
\hline Projeto Básico de Aeronaves & $5^{\mathrm{a}}$ & & & $\mathrm{X}$ & \\
\hline Fundamentos de Circuitos Digitais & $2^{\mathrm{a}}$ & & & & $\mathrm{X}$ \\
\hline Circuitos Analógicos & $2^{\mathrm{a}}$ & & & & $\mathrm{X}$ \\
\hline $\begin{array}{l}\text { Eletrônica Fundamentos } \\
\text { Analógica }\end{array}$ & $3^{\mathrm{a}}$ & & & & $\mathrm{X}$ \\
\hline Controle de Processos & $5^{\mathrm{a}}$ & & & & $\mathrm{X}$ \\
\hline Sistemas de Controle II & $5^{\mathrm{a}}$ & & & & \\
\hline
\end{tabular}

Fonte: dados da pesquisa

Pela análise do Quadro 2 notamos que existe um professor que atua na $1^{\text {a }}$ série, um na $2^{\underline{a}}$ série, um na $3^{\underline{a}}$ série, quatro na $4^{\underline{a}}$ série e todos na $5^{\underline{a}}$ série, ou seja, todos os professores que responderam esse formulário atuavam em disciplinas ministradas nas duas últimas séries, isto é, trabalham em disciplinas específicas do curso. Por atuarem em disciplinas diferentes, conseguimos analisar um número amplo de disciplinas específicas dos cursos de Engenharia Elétrica, Eletrônica, Controle e Automação e Mecânica. As disciplinas de Fundamentos de Circuitos Analógicos e Fundamentos de Circuitos Digitais são oferecidas nos cursos de Engenharia Elétrica, Eletrônica, de Controle e Automação e Computação. As disciplinas Sistema de Controle I, Sistema de Controle II estão presentes nas Engenharias Elétrica, Eletrônica, de Controle e Automação.

Outro ponto importante a ressaltar, com esse primeiro momento da entrevista mais especificamente com essa questão, é que pudemos observar quais disciplinas esses professores atuaram recentemente, tendo dessa forma, a oportunidade de avaliar os planos de ensino para termos uma ideia dos pré-requisitos dessas disciplinas específicas, e com isso ter uma base dos conteúdos de Algebra Linear que poderiam ser explorados nessas disciplinas e como estariam sendo explorados, preparando-me dessa forma para a entrevista realizada no momento posterior.

A resposta a terceira pergunta foi unânime, todos os entrevistados disseram que utilizavam conceitos de Álgebra Linear nas disciplinas em que atuavam.

Por fim, a quarta questão apresenta como resposta no Quadro 3, quais conteúdos de Álgebra Linear os professores participantes desta pesquisa consideram que utilizam nas disciplinas em que atuaram em 2019.

Quadro 3: Conteúdos de AL utilizados nas disciplinas que os professores atuaram em 2019

\begin{tabular}{|l|c|c|c|c|}
\hline \multicolumn{1}{|c|}{ Conteúdo } & Professor 1 & Professor 2 & Professor 3 & Professor 4 \\
\hline Sistemas Lineares & $\mathrm{X}$ & $\mathrm{X}$ & $\mathrm{X}$ & $\mathrm{X}$ \\
\hline Espaços vetoriais sobre os reais & & $\mathrm{X}$ & & \\
\hline Subespaços vetoriais & & & & \\
\hline Dependência Linear: Base e Dimensão & $\mathrm{X}$ & & & \\
\hline Transformações Lineares & $\mathrm{X}$ & $\mathrm{X}$ & & $\mathrm{X}$ \\
\hline
\end{tabular}




\begin{tabular}{|l|c|c|c|c|}
\hline Autovalores e autovetores & $\mathrm{X}$ & & $\mathrm{X}$ \\
\hline Diagonalização & & & $\mathrm{X}$ & \\
\hline Produto Interno & & & & \\
\hline Ortogonalização & & $\mathrm{X}$ & & \\
\hline Outros & & & & $\mathrm{X}$ \\
\hline
\end{tabular}

Fonte: dados da pesquisa

Pela análise do Quadro 3 notamos que nenhum professor utiliza em suas disciplinas os conteúdos de subespaço vetorial e produto interno, existe um professor que utiliza espaços vetoriais sobre o corpo dos reais, um utiliza dependência linear: base e dimensão, um utiliza diagonalização, um utiliza ortogonalização, dois utilizam autovalores e autovetores, três disseram que utilizam transformação lineares e todos utilizam sistemas lineares.

Observamos que o conteúdo mais utilizado é sistemas lineares, seguido por transformações lineares que são conteúdos que foram mais abordados em dissertações e teses de 2011 até 2018 (PHILOT, 2020). O conteúdo de autovalor e autovetor foi abordado pelos professores 1 e 4, porém posteriormente, o professor 1 disse que não utiliza esse conteúdo nas disciplinas que leciona, mas que tem conhecimento que a disciplina de Sistema de Controle II faz o uso deste conceito e que também já utilizou para resolver problemas em sua carreira profissional.

Outro fato que podemos destacar é que embora alguns professores afirmaram que não utilizavam um determinado conteúdo, sabemos que alguns conceitos da Álgebra Linear estão vinculados a este. Por exemplo, o professor 1 aponta o uso dos objetos matemáticos base e dimensão, porém nos informa que não emprega os conceitos de espaço e subespaço vetorial. Este aspecto mereceria um aprofundamento para conhecer como o professor desenvolve em suas aulas o conceito de dependência linear e a real necessidade de se incluir ou não os objetos matemáticos base e dimensão.

\section{CONSIDERAÇÕES FINAIS}

Apresentamos neste artigo um recorte de uma entrevista reflexiva, relatando o primeiro momento, o contato inicial, que nos forneceu um breve panorama dos conteúdos de Álgebra Linear mais abordados nas disciplinas específicas nas quais esses professores atuam. Dessa análise podemos refletir sobre quais os tópicos da Álgebra Linear devem ser enfatizados em um curso oferecido para Engenharia.

Percebemos que a utilização de alguns temas de Álgebra Linear em disciplinas específicas dos cursos de Engenharia surge com mais frequência nos anos finais. Desta forma, podemos conjecturar que o momento mais oportuno para se ofertar a disciplina de Álgebra Linear seria próximo a esta utilização, evitando o esquecimento desses conceitos e proporcionando assim uma maior vinculação. Além desse fato, espera-se que nesse momento o aluno tenha mais maturidade matemática, o que poderia auxiliar na aprendizagem desses conceitos que são abstratos, fato que corrobora com Prado (2016).

Salientamos que o questionário forneceu subsídios para a continuação do trabalho de doutorado em desenvolvimento, uma vez que as informações obtidas por meio dele possibilitou à entrevistadora a familiarização com alguns conceitos das disciplinas dos cursos de Engenharia auxiliando em uma visão geral dos conteúdos. Essas informações também proporcionaram que a entrevistadora pudesse investigar os planos de ensino, livros textos, linguagens e notações utilizadas, facilitando assim a comunicação entre as áreas na entrevista presencial. 
Esses dois momentos da entrevista, deram indícios dos temas da Engenharia nos quais seria possível encontrar aplicações reais dos conceitos de Álgebra Linear para a construção de um evento contextualizado que se utiliza os conteúdos matemático de autovalor e autovetor.

\section{Agradecimentos}

Agradeço a PUC-SP, pelo apoio financeiro e o Instituto Mauá de Tecnologia - IMT, pelo apoio e incentivo.

\section{REFERÊNCIAS}

BRASIL. Ministério da Educação. Resolução CNE/CES n. 2/2019, de 23 de abril de 2019. Institui as Diretrizes Curriculares Nacionais (DCN) do Curso de Graduação em Engenharia, 2019.

CAMARENA, P. Metodología curricular para las ciencias básicas en ingeniería. Revista Innovación Educativa, v. 2, n. 10 e n. 11, p. 22-28 e 4-12, 2002.

CAMARENA, P. Reporte del proyecto de investigación intitulado: Las competencias y calidad de la ingeniería y las ciencias básicas. México: Editorial ESIME-IPN, 2009.

CAMARENA, P. A treinta años de la teoría educativa "Matemática en el Contexto de las Ciencias". Revista Innovación Educativa, v. 13, n. 62, p. 17-44, 2013a.

CAMARENA, P. La matemática social en el desarrollo integral del alumno. Revista Innovación Educativa, v. 14, n. 65, p. 143-149, maio/ago. 2014.

CAMARENA, P. Didáctica de la matemática em contexto. Educ. Matem. Pesq., São Paulo, vol. 19, n.2, 01-26, 2017.

CELESTINO, Marcos R. Ensino-aprendizagem da Álgebra Linear: as pesquisas brasileiras na década de 90. São Paulo, 2000. 113 p. Dissertação (Mestrado em Educação Matemática). Programa de Pós-Graduados em Educação Matemática, Pontifícia Universidade Católica de São Paulo.

FERRUZI, Elaine Cristina; ALMEIDA, Lourdes Maria Werle de. Modelagem Matemática no ensino de Matemática para engenharia. II Jornada Brasileira do Grupo de Pesquisa Euro-Latino-Americano, v. 6, n. 1, p. 153-172, jan-abr, 2013.

FIRMINO, G.L; SIQUEIRA, A.M. A Matemática no ensino de Engenharia. The Journal of Engineering and Exact Sciences -JCEC, v. 3, n.3, p. 331-345, 2017. http://doi.org/10.18540/2446941603032017331

FONTENELE, F. C. F. Contribuições da Sequência Fedathi para o desenvolvimento do Pensamento Matemático Avançado: uma análise da mediação docente em aulas de Álgebra Linear. Ceará, 2018. 192 p. Tese (Doutorado em Educação). Programa de Pós-Graduados em Educação, Universidade Federal do Ceará.

NOMURA, J. I. Esquemas Cognitivos e Mente Matemática inerentes ao objeto matemático autovalor e autovetor : traçando diferenciais na formação do 
Engenheiro. São Paulo, 2014. 349 p. Tese (Doutorado em Educação

Matemática). Programa de Pós-Graduados em Educação Matemática, Pontifícia Universidade Católica de São Paulo.

POOLE, D. Álgebra Linear. São Paulo: Pioneira Thomson Learning, 2006.

PHILOT, J.; BIANCHINI, B. Um panorama das dissertações e teses sobre o tema álgebra linear no ensino superior no Brasil nos anos de 2011 a 2018. Revista de Produção Discente em Educação Matemática, São Paulo, v.9, p. 94-106, 2020.

PRADO, E. de A. Álgebra Linear na Licenciatura em Matemática: contribuições para a formação do profissional da Educação Básica. São Paulo, 2016. 252 p. Tese (Doutorado em Educação Matemática). Programa de Pós-Graduados em Educação Matemática, Pontifícia Universidade Católica de São Paulo.

RIBEIRO, L. N. Uma análise do movimento de constituição da ementa da disciplina de Álgebra Linear na Licenciatura em Matemática. Goiás, 2018. XX p. Dissertação (Mestrado em Educação em Ciências e Matemática). Programa de Pós-Graduados em Educação em Ciências e Matemática, Universidade Federal de Goiás.

SZYMANSKI, Heloiza; ALMEIDA, Laurinda Ramalho; PRANDINI, Regina Célia Almeida Rego. A Entrevista na Pesquisa em Educação a prática reflexiva. Campinas: Editora Autores Associados LTDA. 2018.

\title{
INSTRUCTIONS FOR PREPARATION AND SUBMISSION OF WORKS TO THE SCIENTIFIC COMMITTEE OF XLIX BRAZILIAN CONGRESS OF ENGINEERING EDUCATION - COBENGE 2021
}

\begin{abstract}
Research in Mathematical Education points to the importance of Linear Algebra in several undergraduate courses, especially Engineering. It is a consensus among researchers in this area that students have difficulties in understanding the content covered in Linear Algebra due to the formalism inherent in this subject and the lack of mathematical maturity of students at the time that this discipline is taught in the courses. One aspect that is highlighted in the National Curriculum Guidelines for the Undergraduate Engineering Course is that Engineering courses can no longer be seen as a set of technical, or nontechnical, isolated knowledge, this points out that the contextualization of subjects should be privileged. In this sense, the objective of this article is to present a record of a doctoral research that has as one of its focuses to elaborate contextualized learning situations for the teaching of eigenvalue and eigenvector for Engineering courses. The analysis of a questionnaire answered by professors of specific disciplines of Engineering courses of a Higher Education Institution is presented, with the perspective of understanding what the contents of Linear Algebra are most explored. It is noticed that all the interviewees use in their classes the mathematical object linear systems and that more specific subjects, such as eigenvalue and eigenvector are present in subjects taught at the end of the course.
\end{abstract}

Keywords: Linear Algebra in Engineering, Interview with professors. 